\title{
Journal of Neurodevelopmental Disorders reviewer acknowledgement 2014
}

Joseph Piven

\section{Contributing reviewers}

We thank all of our reviewers who have contributed to the journal in volume 6 (2014). High quality and timely reviews are critical to the overall quality of the journal. We are committed to providing a unique and important outlet for scholarship regarding neurodevelopmental disorders and are indebted to the outstanding reviewers who have contributed their time over the last year in helping us to reach this goal.

Brett Abrahams

United States of America

Jane Adams

United States of America

Maria Arvio

Finland

Torsten Baldeweg

United Kingdom

Simon Baron-Cohen

United Kingdom

Ben A. Barres

United States of America

Renata Bartesaghi

Italy

Ayelet Ben-Sason

Israel

Raphael Bernier

United States of America

Nan Bernstein Ratner

United States of America

Catalina Betancur

France

Dorothy Bishop

United Kingdom

\section{Somer Bishop}

United States of America

Sven Bolte

Sweden

Maria Clara Bonaglia

Italy

Oliver Braddick

United Kingdom

William Brown

United States of America

George Capone

United States of America

Melissa Carter
Canada

Leslie Carver

United States of America

Carissa Cascio

United States of America

Tony Charman

United Kingdom

Abhijit Chaudhuri

United Kingdom

John Colombo

United States of America
Andrea Contestabile
Italy

Gina Conti-Ramsden

United Kingdom

Michael Corballis

New Zealand

Michael Craig

United Kingdom

Steve Dager

United States of America

Kim Dalton

United States of America

Julie Daniels

United States of America

Gabriel Dichter

United States of America

Dagmara Dimitriou

United Kingdom

Magdalena Dziembowska

Poland

Inge-Marie Eigsti

United States of America

Christa Einspieler

Austria

Correspondence: jneurodevdisorders@biomedcentral.com

Carolina Institute for Developmental Disabilities, University of North Carolina,

Chapel Hill, 100 Renee Lynne Court, Carrboro, NC 27510, USA

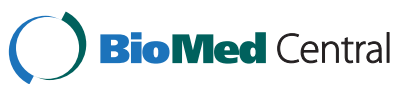

(c) 2015 Piven; licensee BioMed Central. This is an Open Access article distributed under the terms of the Creative Commons Attribution License (http://creativecommons.org/licenses/by/4.0), which permits unrestricted use, distribution, and reproduction in any medium, provided the original work is properly credited. The Creative Commons Public Domain Dedication waiver (http://creativecommons.org/publicdomain/zero/1.0/) applies to the data made available in this article, unless otherwise stated. 
Peggy Eis

United States of America

Jed Elison

United States of America

Susan Faja

United States of America

Emily Farran

United Kingdom

Deborah Fidler

United States of America

Susan Folstein

United States of America

Tim Fosker

United Kingdom

Nathan Fox

United States of America

Nouchine Hadjikhani

United States of America

Eveline Hagebeuk

Netherlands

Brian Hallahan

Ireland

Elizabeth Head

United States of America

Patricia Howlin

United Kingdom

Eric Huang

United States of America

Yadong Huang

United States of America

Lisa M. Jacola

United States of America

Shafali Jeste

United States of America

Michael V. Johnston

United States of America

Robert Joseph

United States of America

Rajesh Kana

United States of America

Katherine Karlsgodt

United States of America

Walter Kaufmann

United States of America
Kelly King

United States of America

Bonita Klein-Tasman

United States of America

Kami Koldewyn

United Kingdom

Nikolaos Kozeis

Greece

Silvia Lanfranchi

Italy

Hayley Leonard

United Kingdom

April Levin

United States of America

Ovsanna Leyfer

United States of America

Molly Losh

United States of America

Eva Loth

United Kingdom

Beth Malow

United States of America

Christian Marshall

Canada

Peter Marshall

United States of America

Karla McGregor

United States of America

Carolyn Mervis

United States of America

Paul Moes

United States of America

Anna Victoria Molofsky

United States of America

Eric Morrow

United States of America

Matt Mosconi

United States of America

Kazuhiko Nakabayashi

Japan

Masato Nakafuku

United States of America

Giovanni Neri

Italy
Nora Newcombe

United States of America

Craig Newschaffer

United States of America

Rowena Ng

United States of America

Rob Nicolson

Canada

Jose Luis Olmos-Serrano

United States of America

Lucy Osborne

Canada

Opal Ousley

United States of America

Tyler Perrachione

United States of America

Isaac Pessah

United States of America

Karen Pierce

United States of America

Elena Plante

United States of America

Daniela Plesa-Skwerer

United States of America

Melanie Porter

Australia

John R. Pruett Jr

United States of America

Zhenghan Qi

United States of America

Michael Rafii

United States of America

Susan Ravizza

United States of America

Keith Rayner

United States of America

Francesco Ria

Italy

Mabel Rice

United States of America

Jane Roberts

United States of America

Timothy Roberts

United States of America 
Randal Ross

United States of America

Joanne Rovet

Canada

Fabio Sambataro

Italy

Carolyn Schanen

United States of America

Stephen Scherer

Canada

Suzy Scherf

United States of America

Michael Schlund

United States of America

Sarah Schoen

United States of America

Robert Schultz

United States of America

Elsa Shapiro

United States of America

Frederick Shic

United States of America

Tony Simon

United States of America

Karun Singh

Canada
Liz Smith

United Kingdom

Jesse Snedeker

United States of America

Mikle South

United States of America

Sarah Spence

United States of America

Daniel Stahl

United Kingdom

Catherine Stamoulis

United States of America

Julia Stephen

United States of America

Courtney Stevens

United States of America

Yujiao Sun

United States of America

John Sweeney

United States of America

Frank Symons

United States of America

Nicole Tartaglia

United States of America

Michel Thiebaut de Schotten

United Kingdom
Brent Vander Wyk

United States of America

Stefano Vicari

Italy

Giacomo Vivanti

Australia

Jennifer Wagner

United States of America

Seth Warschausky

United States of America

Sara Webb

United States of America

Corrine Welt

United States of America

Andrew Whitehouse

Australia

Kate Woodcock

United Kingdom

Timothy Yu

United States of America

Ryan Yuen

Canada 